\section{Long term follow-up of benign functional adrenocortical tumors in the era of robotic surgery}

\author{
Martin Nilsson, Martin Almquist, \\ Erik Nordenström
}

Department of Surgery, Skane University Hospital, Lund University, Lund, Sweden

\section{Abstract}

Long-term outcome after operation for benign functional adrenal tumours [aldosteronoma (PA), subclinical (SCS) or clinical Cushing's syndrome (CS)] has not been reported extensively, especially not in the era of roboticassisted laparoscopic surgery why pheochromocytomas has not been included in the group of benign functional masses? We have excluded pheocromocytoma because it is well known that it is very difficult to distinguish between malignant and benign pheocromocytomas. Since our aim was to evaluate benign lesions, we excluded pheocromocytomas. The purpose of this study was to investigate the clinical outcome for patients having undergone adrenalectomy for non-malignant clinical or subclinical functional adrenocortical tumors. The records of 65 patients with benign functional adrenocortical tumors treated with laparoscopic adrenalectomy (80\% robotic assisted) were surveyed retrospectively. Clinical and biochemical data were evaluated at baseline and followup data was collected by a survey distributed to referring endocrinology centers. Cure was defined both in objective measurements and in the subjective validation of the endocrinologists referring the patients for surgery. According to our definition, $83 \%$ of patients with PA were either cured (normotension without anti-hypertensive medication), or improved (better blood pressure control with equal or fewer medications). For CS, $89 \%$ of patients were cured (biochemical normalization and lack of or significant improvement in co-morbidities). With the same definition, $73 \%$ of SCS patients were cured. The endocrinologists considered $86 \%$ of PA patients, $100 \%$ of CS and $64 \%$ of SCS patients as cured. Complications were few and there was no mortality. Robotic assisted adrenalectomy provides safe and effective treatment for PA, CS and SCS. Our objective definition of cure matched the assessment of cure of the endocrinologists in patients with PA and CS.

\section{Introduction}

Patients with functional benign adrenocortical tumours [i.e. aldosteronomas, unilateral subclinical (SCS) or clinical (CS) Cushing's disease] are often recommended resection.1,2 Success of surgical treatment varies widely, however, with studies showing cure rates ranging from $27 \%$ to $82 \%$ for aldosteronomas and up to $99 \%$ for Cushing syndrome..$^{3-7}$ Surgery for SCS has shown improvement of hypertension, body mass index (BMI) and hyperlipidemia. 8

With the introduction of laparoscopic techniques for adrenalectomy, and recently also robotic-assisted devices, the frequency of adrenalectomies has increased markedly. ${ }^{9}$ Hence, it is likely that adrenalectomy today is performed more liberally than previously, which could affect the risk/benefit ratio.

Even though many previous studies have suggested that adrenalectomy for benign functional tumours is safe and has good short-term cure rates, reports on outcome after adrenalectomy in the era of robotic surgery are scarce, especially regarding long-term follow up.

It is also interesting to compare objective biologically parameters with the subjective perception of the endocrinologist regarding cure of the patients.

Thus, our aim of the present study was to investigate the clinical outcome and possible benefit for patients having undergone surgery for non-malignant clinical or subclinical functional adrenocortical tumors.

\section{Materials and Methods}

\section{Patients}

One hundred fifty patients who underwent adrenalectomy at the Department of Surgery, Lund, Sweden, between 2000 and 2009 were eligible for the present study. Data were analyzed retrospectively. Inclusion criteria were benign functional adrenocortical tumors: i) elevated aldosterone-renin ratio and unilateral mass or lateralization on adrenal venous sampling (aldosterone producing adenoma or primary adrenal hyperplasia, primary aldosteronism), ii) pathological dexamethasone suppression test and clinical findings suggestive of cortisol excess (adrenal Cushing's syndrome) or iii) pathological dexamethasone suppression test without clinical findings suggestive of cortisol excess (subclinical adrenal Cushing's syndrome). Patient records were investigated for diagnosis and histopathology. Sixty-five patients with benign functional adrenocortical tumors were identified: 40 patients with Conn's disease [primary aldos-
Correspondence: Erik Nordenström, Department of Surgery, Skane University Hospital, Lund; Lund University, S-221 85 Lund, Sweden.

Tel.+46.46.172305 - Fax: +46.46 .172335$

E-mail: erik.nordenstrom@skane.se

Key words: adrenocortical tumor, cushing syndrome, primary aldosteronism, laparoscopic adrenalectomy, robotic surgery.

Acknowledgments: we would like to thank the participating endocrinology centers in Lund, Helsingborg, Kristianstad, Växjö, Halmstad, Ängelholm, Varberg, Malmö, Hässleholm and Ljungby, without the interest and help from which the study would not have been possible to realize. Anders Isaksson of the Department of Clinical Chemistry, Lund helped us out with the analytic differences between regional laboratory facilities.

Conflict of interests: the authors report no conflict of interests.

Received for publication: 4 June 2012.

Accepted for publication: 24 July 2012.

This work is licensed under a Creative Commons Attribution NonCommercial 3.0 License (CC BYNC 3.0)

(C) Copyright M. Nilsson et al., 2012

Licensee PAGEPress, Italy

Endocrinology Studies 2012; 2:e4

doi:10.4081/es.2012.e4

teronism (PA)], 11 patients with $\mathrm{CS}$ and 15 with SCS. The patients had been referred for surgery from ten endocrinology centers in Southern Sweden.

\section{Baseline data and definitions}

Preoperative data were collected and entered into the online Scandinavian quality register for Thyroid and Parathyroid Surgery incorporating Adrenal Surgery. Data included gender, age, detection (incidental versus symptomatic), systolic blood pressure (SBP), diastolic blood pressure (DBP), anti-hypertensive treatment, hypokalemia (serum potassium $<3.2$ $\mathrm{mmol} / \mathrm{L}$ ), potassium regulating treatment (aldosterone receptor antagonist or potassium replacement), weight gain last three months, BMI, diabetes mellitus, densitometry verified osteoporosis, radiological estimated tumor size, non-contrast enhanced attenuation, aldosterone-renin ratio for PA (with a threshold equivalent of $<55 \mathrm{pmol} / \mathrm{mIE}$ ), morning cortisol and ACTH for CS and SCS, outcome of dexamethasone suppression test (with a threshold of $<70 \mathrm{nmol} / \mathrm{L}$ for $1 \mathrm{mg}$ overnight test), serum DHEAS (reference women 1,0$11.7 \mu \mathrm{mol} / \mathrm{L}$ and men $2.2-15.2 \mu \mathrm{mol} / \mathrm{L}$ ), $24 \mathrm{~h}$ urine free cortisol (reference $38-170 \mathrm{nmol} / \mathrm{d}$ ) and urine steroid profile. Hypertension was 
defined by blood pressure (BP) $>140 \mathrm{mmHg}$ systolic and $>90 \mathrm{mmHg}$ diastolic and normotension by a BP $<140 \mathrm{mmHg}$ systolic and $<90 \mathrm{mmHg}$ diastolic.

CT of the abdomen was the imaging modality of choice.

\section{Follow-up}

A survey was sent to all ten referring endocrinology centers comprising questions regarding clinical cure as estimated by clinician, blood pressure, weight and length at last examination, medical treatment for hypertension, improvement in preoperatively diagnosed diabetes mellitus or hyperlipidemia, result of $1 \mathrm{mg}$ dexamethasone suppression test, duration or present dose of hydrocortisone replacement, last measures of electrolytes, aldosterone-renin ratio, morning cortisol and ACTH as well as postoperative diagnosis of cardiovascular disease, diabetes mellitus, hypercholesterolemia, gout or osteoporosis. Supplementary follow-up data was extracted from the electronic patient records if possible. Follow-up time was defined as the duration from date of surgery to last biochemical evaluation and mean follow-up time was 22 months. One patient with CS was lost at follow-up and one patient with CS was excluded due to short follow-up time (1 month). The total response frequency was $97 \%$.

\section{Outcome}

For PA, cure was defined as biochemical normalization and normotension without antihypertensive medication; improvement as equal BP with fewer anti-hypertensive medications or lower BP with equal number of antihypertensive medications. For CS and SCS, cure was defined as biochemical normalization with significant improvement in or absence of co-morbidities (i.e. hypertension, cardiovascular disease, diabetes mellitus, hyperlipidemia and osteoporosis).

\section{Surgical technique}

From the beginning of the study period until December 2005, the standard procedure in Lund was transperitoneal laparoscopic adrenalectomy. Thereafter, robotic assisted transperitoneal laparoscopic adrenalectomy using a $d a$ Vinci $^{\mathrm{TM}}$ Robotic Surgical System (Intuitive Surgical, Sunnyvale, CA, USA) has been the procedure of choice. ${ }^{10}$

\section{Statistics}

Statistical analysis was performed using Stata 11/IC (StataCorp LP, College Station, TX, USA). Continuous and discrete variables were analyzed using matched Student's t-test and Wilcoxon signed-rank test, as applicable. For analysis of ACTH, logarithmic transformation in conjunction with Student's t-test was per- formed. Group comparisons were carried out using Kruskal-Wallis one-way analysis of variance and Mann-Whitney U-test respectively. Categorical variables were analyzed using McNemar's test and Fisher's exact test. P values $\leq 0.05$ were considered significant. All tests were two-sided.

\section{Ethical considerations}

The Ethical Committee of the Medical Faculty, Lund University, Sweden approved the study.

\section{Results}

\section{Baseline data}

Preoperative data of the 65 patients is described in Table 1. One patient diagnosed with PA postoperatively developed adrenal insufficiency with a deranged hypothalamicpituitary-adrenal-axis, thus qualifying for the diagnosis of SCS. Dexamethasone suppression test had not been performed preoperatively. This patient was included in both groups. Three patients with PA had no radiologically discernible tumor. Adrenal venous sampling was performed in $80 \% \mathrm{PA}, 27 \% \mathrm{CS}$ and $40 \%$ SCS of subjects, respectively. Serum DHEAS was only performed in 22 patients. Urine free cortisol was only assessed in eight of the patients with PA. Investigation for pheochromocytoma such as urine and plasma catecholamines, VMA, chromogranin A, clonidine suppression test or MIBG scintigraphy was carried out in 50\% PA, 64\% CS and 100\% SCS.

\section{Surgery, histopathology and complications}

Surgical characteristics of each group are presented in Table 2 . The great majority of procedures were carried out by unilateral robotic assisted laparoscopy. Conversion occurred in six cases, in three cases caused by difficult access, in two cases by adhesion and in one case by bleeding and difficult access. As the difficulty in same contributed to a higher conversion rate. Our access during robotic assisted adreanlectomy is: the patient was placed in a lateral decubitus position with the adrenal lesion side up. Pneumoperitoneum was created with open technique with a 12 -mm blunt tip trocar for the camera. Two 8-mm trocars were then placed and connected to the left and right robot arms. Finally, one (left side) or two (right side) 12-mm accessory trocars for suction, irrigation and retraction by the first assistant were placed. Hydrocortisone replacement therapy was given to patients undergoing surgery for CS and SCS, as well as to the patient primarily diagnosed with PA post-operatively developing adrenal insufficiency. In eight patients histopathology revealed adrenocortical hyper- plasia and in one subject with clinically evident PA normal adrenal gland, probably microadenoma (Table 2).

Complications were few: in a 73 year old female patient with SCS intensive care unit management was required postoperatively, in a 78 year old female with SCS, bleeding occurred requiring transfusion, and a 54 year old male patient suffered from a subfrenical abscess, however not necessitating surgical treatment.

\section{Follow-up}

Follow-up characteristics are described in Table 3 . The only parameter differing significantly between the groups is present hydrocortisone replacement dose $(\mathrm{P}=0.0052)$.

Endpoint parameters are outlined in Tables 46. Post-operative BMI was only available for 38 patients and dexamethasone suppression test had only been performed after surgery in three patients with CS and seven with SCS.

In the PA group, significant differences post adrenalectomy were found for SBP, DBP, medically treated hypertension, number of antihypertensive agents, hypokalemia, potassium regulation therapy and aldosterone-renin ratio. On the contrary, in the CS group, only the number of anti-hypertensive agent and ACTH differed significantly. Further, in the SCS group, SBP, ACTH, cortisol and incomplete dexamethasone suppression differed significantly. Two patients with PA had cerebrovascular insults postoperatively, whereas one patient was diagnosed with a thoracic aortic aneurysm and another with atrial fibrillation. One patient with PA developed diabetes mellitus type II, and another impaired glucose tolerance. In the SCS group, one patient had a deep vein thrombosis, while there were no reported postoperative diagnoses in the CS group.

Preoperatively diagnosed diabetes mellitus were improved in all three cases of the CS group, ranging from complete withdrawal of insulin to markedly improved $\mathrm{HbA}_{1 \mathrm{c}}$ and normalization of fasting plasma glucose. In the SCS, one patient improved in fasting plasma glucose, for the PA patient no information was available. Regarding hyperlipidemia, one CS patient was able to quit simvastatin medication, and one SCS patient improved on without dose change. For one SCS patient, bone densitometry improved from osteopenia to normal densitometry. According to the Swedish civil registry, there was no mortality in either group up to August 2010.

\section{Outcomes}

Matching of the assessment of cure of the endocrinologist (considered cure) and our objective definition of cure/improvement is showed in Table 7.

According to our definition, $35 \%$ of patients having undergone adrenalectomy for PA were 
cured, and further $48 \%$ improved in either SBP, DBP or in the number of anti-hypertensive medications. The endocrinologists answering the survey considered $86 \%$ of the patients with primary hyperaldosteronism to be clinically cured. In the CS group, $89 \%$ of the patients were cured according to our definition, and in the survey $100 \%$ of the patients with CS was considered as cured. $64 \%$ of the patients with SCS were considered cured by the endocrinologists, while $73 \%$ fulfilled our definition in the SCS group.

\section{Discussion}

In the present cohort of 65 patients having undergone adrenalectomy for benign adrenocortical functional tumors, we found a cure rate of PA, CS and SCS of 35\%, 89\%, and 73\% respectively. Complications to surgery were few, making robotic assisted laparoscopic adrenalectomy a safe choice of treatment. Our results are in line with previous reports. Regarding PA, Pang et al. found a cure rate of $34 \%$, with a median follow-up 59 months in median. ${ }^{3}$ Meria et al. reported a cure rate of 58\% in 212 patients with PA (median follow-up 44 months) and Ku et al. retrospectively found a cure rate of $82 \%$ for PA (median follow-up 11 months). 4,9

Lack of complete resolution of hypertension in PA might be predicted by duration of hypertension and degree of vascular remodeling. ${ }^{11}$ Thus, comparing the series of Ku et al. with our material, their patients were ten years younger (42 in median) and might therefore have had a shorter preoperative duration of hypertension. Unfortunately, it has not been possible to ascertain duration of hypertension in our material. The diagnosis was confirmed on pathology in $95 \%$ of cases.

Comparing cure rates in CS and SCS between studies is more difficult due to differences in definition. Generally, biochemical normalization, regression of hypercortisolism related symptoms and improvement in important comorbidities such as diabetes mellitus, obesity, hypertension, hyperlipidemia and osteoporosis are deemed important. Thus, one report found four of eight patients to respond well to surgery, ${ }^{5}$ while another found four of five patients to be biochemically normalized and to lack any sign or symptom of hypercortisolism. ${ }^{6} \mathrm{Ku}$ et al . found resolvement or improvement of hypercortisolism symptoms in all 15 patients at follow-up, and that SBP, DBP and the number of anti-hypertensive agents were significantly reduced. ${ }^{8}$ In a recent retrospective study comparing outcome for CS and SCS, Alesina et al. found that their 71 SCS patients were significantly more often biochemically normalized, but only CS patients $(\mathrm{n}=99)$ were significantly
Table 1. Baseline characteristics of 65 patients having undergone surgery for functional adrenocortical tumors in Lund 2000-2009.

\begin{tabular}{lcccc} 
& PA (no.) & CS (no.) & SCS (no.) & Total (no.) \\
N. & 40 & 11 & 15 & 65 \\
Gender, M : F & $24: 16$ & $2: 9$ & $1: 14$ & $27: 38$ \\
\hline Age, years & $52.3 \pm 10.2$ & $52.7 \pm 9.99$ & $61.7 \pm 10.6$ & $54.7 \pm 10.8$ \\
Radiological tumor size, mm* & $15,11-20$ & $35,28-45$ & $40,35-50$ & $25,15-35$ \\
\hline Tumor side, left-right-bilateral & $27-12-1$ & $3-5-3$ & $9-2-4$ & $38-19-8$ \\
Attenuation, HU* & $5,-6-10$ & $14.5,2.5-21.5$ & $6,-1-10$ & $5.5,0-12$ \\
\hline Incidental detection & $7.5 \%(3)$ & $36 \%(4)$ & $87 \%(13)$ & $31 \%(20)$ \\
Hypokalemia & $95 \%(38)$ & $36 \%(4)$ & $6.7 \%(1)$ & $65 \%(42)$ \\
\hline Weight gain last 3 months & $10 \%(4)$ & $36 \%(4)$ & $13 \%(2)$ & $15 \%(10)$ \\
Diabetes mellitus & $2.5 \%(1)$ & $27 \%(3)$ & $6.7 \%(1)$ & $7.7 \%(5)$ \\
\hline Osteoporosis & $0 \%$ & $36 \%(4)$ & $20 \%(3)$ & $11 \%(7)$ \\
DHEAS suppressed & $0 \%$ & $55 \%(6)$ & $67 \%(10)$ & $25 \%(16)$ \\
\hline Urine cortisol increased & $2.5 \%(1)$ & $73 \%(8)$ & $6.7 \%(1)$ & $15 \%(10)$ \\
Urine steroid profile abnormal & $5 \%(2)$ & $55 \%(6)$ & $27 \%(4)$ & $17 \%(11)$ \\
\hline
\end{tabular}

Data mean \pm standard deviation. PA, primary aldosteronism; CS, cushing syndrome; SCS, subclinical cushing syndrome. * Median, interquartile range.

Table 2. Surgical characteristics of 65 patients having undergone surgery for functional adrenocortical tumors in Lund 2000-2009.

\begin{tabular}{lcccc} 
& PA (no.) & CS (no.) & SCS (no.) & Total (no.) \\
No. & 40 & 11 & 15 & 65 \\
Unilateral-bilateral & $39-1$ & $9-2$ & $15-0$ & $62-3$ \\
\hline Open-laparoscopic-robotic assisted & $5-6-29$ & $1-1-9$ & $1-0-14$ & $6-7-52$ \\
Conversion to open surgery & $13 \%(5)$ & $0 \%$ & $6.7 \%(1)$ & $9.2 \%(6)$ \\
\hline Post-operative days at ward* & $3,3-4$ & $7,6-7$ & $4,3-7$ & $4,3-7$ \\
Hydrocortisone replacement & $2.5 \%(1)$ & $91 \%(10)$ & $87 \%(13)$ & $35 \%(23)$ \\
\hline Adenoma on histopathology & $95 \%(38)$ & $82 \%(9)$ & $67 \%(10)$ & $86 \%(56)$ \\
\hline
\end{tabular}

Data mean \pm standard deviation. PA, primary aldosteronism; CS, cushing syndrome; SCS, subclinical cushing syndrome. *Median, interquartile range.

Table 3. Follow-up characteristics of 65 patients having undergone surgery for functional adrenocortical tumors in Lund 2000-2009.

\begin{tabular}{lcccc} 
& PA (no.) & CS (no.) & SCS (no.) & Total (no.) \\
No. & 40 & 9 & 15 & 63 \\
Gender, M:F & $24: 16$ & $2: 7$ & $1: 14$ & $27: 36$ \\
\hline Follow-up time, months* & $22,12-35$ & $24,19-61$ & $22,10-31$ & $22,13-33$ \\
Hydrocortisone replacement, months* & 0 & $23,14-43$ & $1.9,0-13$ & $10,0-23$ \\
\hline Hydrocortisone replacement, mg/day & 0 & $28 \pm 13$ & $15 \pm 7.1$ & $23 \pm 12$ \\
Considered cured by clinician & $78 \%(31)$ & $89 \%(8)$ & $53 \%(8)$ & $73 \%(46)$
\end{tabular}

Data mean \pm standard deviation. PA, primary aldosteronism; CS, cushing syndrome; SCS, subclinical cushing syndrome. *Median, interquartile range.

Table 4. Endpoint parameters of 40 patients having undergone surgery for Conn's disease.

\begin{tabular}{lccc} 
& Preoperative (no.) & Follow-up (no.) & P value \\
SBP, $\mathrm{mm} \mathrm{Hg}$ & $154 \pm 13.9$ & $134 \pm 14.9$ & $<0.0001(\mathrm{n}=40)$ \\
DBP, $\mathrm{mm} \mathrm{Hg}$ & $89.9 \pm 10.3$ & $82.3 \pm 9.62$ & $0.0001(\mathrm{n}=40)$ \\
\hline medically treated hypertension & $100 \%$ & $58 \%(23)$ & $<0.0001(\mathrm{n}=40)$ \\
anti-hypertensive agents, $\mathrm{n}$ & $2.8 \pm 1.3$ & $1.3 \pm 1.2$ & $<0.0001(\mathrm{n}=40)$ \\
\hline hypokalemia & $95 \%(38)$ & $0 \%$ & $<0.0001(\mathrm{n}=40)$ \\
K regulation & $95 \%(38)$ & $0 \%$ & $<0.0001(\mathrm{n}=34)$ \\
\hline Body mass index, $\mathrm{kg} / \mathrm{m}^{2}$ & $28.4 \pm 4.72$ & $27.4 \pm 4.15$ & $0.1897(\mathrm{n}=21)$ \\
Aldosterone-renin ratio, $\mathrm{pmol} / \mathrm{mIE}^{*}$ & $175,116-283$ & $9.36,6.25-20.8$ & $<0.0001(\mathrm{n}=27)$ \\
\hline
\end{tabular}

SBP, systolic blood pressure; DBP, diastolic blood pressure. Data mean \pm standard deviation. *Median, interquartile range. 
normalized in BMI, hypertension and number of anti-hypertensive agents after adrenalectomy. ${ }^{7}$ In the only randomized trial available for SCS by Toniato et al., improvement or normalization after surgery was suggested in diabetes mellitus, obesity and hypertension, though significant only in the latter. ${ }^{12}$ Chiodini et al. similarly found their SCS patients $(n=25)$ having undergone surgery improved more frequently in weight, blood pressure and glucose than patients on conservative treatment, and Guerrieri et al. found their surgically treated patients $(n=19)$ to improve significantly in BMI, SBP, DBP, oral glucose tolerance test and HDL cholesterol.13,8 However, the data available don't allow determination of their respective cure rate according to our definition.

Differences in outcome after surgery probably reflect factors such as study design, number of patients included, selection of patients to the study clinic, choice of measures (e.g. fasting glucose $v s$ oral glucose tolerance test etc). How can cure be defined in asymptomatic disease, and is it useful? In the present study this is

Table 5. Endpoint parameters of 9 patients having undergone surgery for Cushing's syndrome.

\begin{tabular}{lccc} 
& Preoperative & Follow-up (no.) & P value \\
SBP, $\mathrm{mm} \mathrm{Hg}$ & $151 \pm 27.9$ & $140 \pm 22.7$ & $0.4060(\mathrm{n}=9)$ \\
DBP, mm Hg & $85.5 \pm 18.1$ & $83.9 \pm 8.04$ & $0.7165(\mathrm{n}=9)$ \\
\hline Medically treated hypertension & $100 \%$ & $89 \%(8)$ & $1.0000(\mathrm{n}=9)$ \\
Anti-hypertensive agents, $\mathrm{n}$ & $3.0 \pm 1.3$ & $1.8 \pm 1.1$ & $0.0400(\mathrm{n}=9)$ \\
\hline Body mass index, $\mathrm{kg} / \mathrm{m}^{2}$ & $26.9 \pm 3.01$ & $25.3 \pm 5.01$ & $0.1769(\mathrm{n}=6)$ \\
ACTH, 8 AM pmol/L* & $0.23,0.23-0.63$ & $3.8,2.3-5.7$ & $0.0008(\mathrm{n}=5)$ \\
\hline Cortisol, 8 AM nmol/L & $678 \pm 255$ & $340 \pm 232$ & $0.0759(\mathrm{n}=7)$ \\
Incomplete dexamethasone suppression & $100 \%$ & $11 \%(1)$ & $0.5000(\mathrm{n}=3)$ \\
\hline
\end{tabular}

SBP, systolic blood pressure; DBP, diastolic blood pressure. Data mean \pm standard deviation. *Median, interquartile range.

Table 6. Endpoint parameters of 15 patients having undergone surgery for subclinical Cushing's syndrome.

\begin{tabular}{lccc} 
& Preoperative & Follow-up (no.) & P value \\
SBP, $\mathrm{mm} \mathrm{Hg}$ & $146 \pm 17.7$ & $129 \pm 16.0$ & $0.0016(\mathrm{n}=15)$ \\
DBP, $\mathrm{mm} \mathrm{Hg}$ & $83.6 \pm 6.93$ & $79.5 \pm 7.22$ & $0.0629(\mathrm{n}=15)$ \\
\hline Medically treated hypertension & $67 \%(10)$ & $67 \%(10)$ & $1.0000(\mathrm{n}=15)$ \\
Anti-hypertensive agents & $1.4 \pm 1.3$ & $1.2 \pm 1.1$ & $0.3007(\mathrm{n}=15)$ \\
\hline BMI, $\mathrm{kg} / \mathrm{m}^{2}$ & $25.9 \pm 4.09$ & $24.7 \pm 3.28$ & $0.4749(\mathrm{n}=12)$ \\
ACTH, $8 \mathrm{AM} \mathrm{pmol} / \mathrm{L}^{*}$ & $0.95,0.23-2.0$ & $5.6,1.8-12$ & $0.0009(\mathrm{n}=12)$ \\
\hline Cortisol, $8 \mathrm{AM} \mathrm{nmol} / \mathrm{L}$ & $550 \pm 176$ & $442 \pm 172$ & $0.0093(\mathrm{n}=14)$ \\
Incomplete dexamethasone suppression & $87 \%(13)$ & $7.1 \%(1)$ & $0.0313(\mathrm{n}=7)$ \\
\hline
\end{tabular}

SBP, systolic blood pressure; DBP, diastolic blood pressure. Data mean \pm standard deviation. ${ }^{*}$ Median, interquartile range.

Table 7. Matching of the assessment of cure (considered cure) of the endocriniologist and our objective definition of cure in patients operated on for primary aldosteronism, Cushing syndrome and Subclinical cushing syndrome.

Considered cured Not consider cured

Primary aldosteronism

Cured/improved $(\mathrm{n}=33)$

Not cured $(n=7)$

Total

Cushing syndrome

Cured/improved ( $\mathrm{n}=8)$

Not cured $(\mathrm{n}=1)$

Total

Subclinical cushing syndrome

Cured/improved $(\mathrm{n}=11)$

Not cured $(\mathrm{n}=4)$

Total

$\begin{array}{cc}31 & 2 \\ 0 & 7 \\ 31 & 9 \\ & \\ 8 & 0 \\ 0 & 1 \\ 8 & 1\end{array}$

8

8

0

8

\section{Conclusions}

There are some limitations of this study. First of all, this, this is a retrospective single-center study. Thus, all data was collected from patient records which means that all measurements were made in everyday clinical practice and data might thus be misclassified. The singlecenter nature implies a possible selection bias. Another weakness is that no patient related outcome measurements i.e. life quality validation were used. Further studies regarding outcome after adrenal surgery for adrenocortical adenomas would be of most interest. Especially studies evaluating outcome and matching the three compounds of objective measurements such as blood pressure and biochemical variables, subjective assessment of the endocriniologist endocrinologist regarding cure/ improvement and life quality before and after surgery experienced by the patient are warranted.

\section{References}

1. Sukor N. Endocrine hypertension - current understanding and comprehensive management review. Eur J Intern Med 2011;22: 433-40.

2. Mulatero $\mathrm{P}$, Monticone $\mathrm{S}$, Veglio $\mathrm{F}$. Diagnosis and treatment of primary aldosteronism. Rev Endocr Metab Disord 2011; 12:3-9.

3. Pang TC, Bambach C, Monaghan JC, et al. Outcomes of laparoscopic adrenalectomy for hyperaldosteronism. ANZ J Surg 2007; 77:768-73.

4. Meria P, Kempf BF, Hermieu JF, et al. Laparoscopic management of primary hyperaldosteronism: clinical experience with 212 cases. J Urol 2003;169:32-5.

5. Ross EJ, Linch DC. The clinical response to treatment in adult Cushing's syndrome following remission of hypercortisolaemia. 
Postgrad Med J 1985;61:205-11.

6. Brunt LM, Moley JF, Doherty GM, et al. Outcomes analysis in patients undergoing laparoscopic adrenalectomy for hormonally active adrenal tumors. Surgery 2001: 130:629-34.

7. Alesina PF, Hommeltenberg S, Meier B, et al. Posterior retroperitoneoscopic adrenalectomy for clinical and subclinical Cushing's syndrome. World J Surg 2010; 34:1391-7.

8. Guerrieri M, Campagnacci R, Patrizi A, et al. Primary adrenal hypercortisolism: minimally invasive surgical treatment or medical therapy? A retrospective study with long-term follow-up evaluation. Surg Endosc 2010;24:2542-6.

9. Ku JH, Yeo WG, Kwon TG, Kim HH. Laparoscopic adrenalectomy for functioning and non-functioning adrenal tumors: analysis of surgical aspects based on histological types. Int J Urol 2005;12:1015-21.

10. Nordenström E, Hallgrimsson $P$, Bergenfelz A. A prospective study of 100 robotically assisted laparoscopic adrenalectomies. Journal of Robotic Surgery 2011;5:127-31.

11. Rossi GP, Bolognesi M, Rizzoni D, et al. Vascular remodeling and duration of hypertension predict outcome of adrena- lectomy in primary aldosteronism patients. Hypertension 2008;51:1366-71.

12. Toniato A, Merante-Boschin I, Opocher G, et al. Surgical versus conservative management for subclinical Cushing syndrome in adrenal incidentalomas: a prospective randomized study. Ann Surg 2009;249:388-91.

13. Chiodini I, Morelli V, Salcuni AS, et al. Beneficial metabolic effects of prompt surgical treatment in patients with an adrenal incidentaloma causing biochemical hypercortisolism. J Clin Endocrinol Metab 2010; 95:2736-45. 